\title{
Physiological roles of leupeptin and extracellular proteases in mycelium development of Streptomyces exfoliatus SMF13
}

\author{
In Seop Kim and Kye Joon Lee
}

\begin{abstract}
Author for correspondence: Professor Kye Joon Lee. Tel: +82 2880 6705. Fax: +82 2882 9285/888 4911. e-mail: 1kj12345@alliant.snu.ac.kr
\end{abstract}

Department of

Microbiology, College of Natural Science, and

Research Centre for Molecular Microbiology, Seoul National University Seoul 151-742, Korea

\begin{abstract}
Streptomyces exfoliatus SMF13 produced leupeptin, chymotrypsin-like protease (CTP), metalloprotease, and trypsin-like protease (TLP) extracellularly. The activity of TLP was specifically inhibited by leupeptin. Production of leupeptin was closely associated with growth but leupeptin was inactivated by leupeptin-inactivating protein (LIP) when growth reached the stationary phase in submerged cultures, or when aerial mycelia started to form on surface cultures. Autolysis of mycelia after the stationary phase in submerged cultures was apparently retarded by the addition of leupeptin; on surface cultures, aerial mycelium formation was clearly retarded by the addition of leupeptin. We propose that CTP participates primarily in utilization of a proteinaceous nitrogen source, that TLP functions as an essential enzyme involved in the metabolism of mycelial protein, that leupeptin inhibits the activity of TLP and that LIP inactivates leupeptin. The cascade of regulatory actions of the compounds, which are produced sequentially during mycelium development, may provide selective advantages in adverse culture conditions.
\end{abstract}

Keywords: Streptomyces exfoliatus, leupeptin, trypsin-like protease, leupeptininactivating protein, morphological differentiation

\section{INTRODUCTION}

Extracellular proteases produced in actinomycetes participate in the assimilation of extracellular proteinaceous nitrogen sources (Shapiro, 1989). Some intracellular proteases take part in the endogenous turnover of cellular proteins and in the processing or post-translational modification of proteins (Bond \& Butler, 1987; Bradley, 1988). Serine protease expressed in stationary phase cultures of Streptomyces peucetius and Streptomyces lactamdurans regulate the cellular turnover metabolism associated with secondary metabolism and morphogenesis (Gibb \& Strohl, 1988; Ginther, 1978). The activity of the regulatory protease may be regulated by an autogenous protease inhibitor (Aoyagi, 1989; Gräfe, 1989). Protease activity associated with sporulation in Coccidioides immitis was regulated by an intracellular protease inhibitor

Abbreviations: BAPNA, $\mathrm{N}$-benzoyl-arginine $p$-nitroanilide; BTPNA, $\mathrm{N}$ benzoyl-tyrosine $p$-nitroanilide; CTP, chymotrypsin-like protease; LIP, leupeptin-inactivating protein; MTP, metalloprotease; PFLNA, p-Glu-PheLeu $p$-nitroanilide; TLCK, Na-p-tosyl-L-lysyl chloromethyl ketone; TLP, trypsin-like protease; TPCK, $\mathrm{N}$-tosyl-t-phenylalanine chloromethyl ketone.
(Yuan \& Cole, 1989), as was an intracellular protease expressed during the transition from vegetative growth to spore formation in Saccharomyces cerevisiae (Holzer, 1983).

Streptomyces spp. produce various forms of protease inhibitors (Umezawa, 1982; Aoyagi, 1989). Leupeptin (Aoyagi et al., 1969), antipain (Suda et al., 1972), chymostatin (Umezawa et al., 1970) and elastatinal (Umezawa et al., 1973) are the best-known products and some may have clinical applications (Aoyagi et al., 1977; Umezawa, 1988). Although progress has been made in the biochemical characterization of proteases and protease inhibitors in Streptomyces spp., much less has been learned of the biological roles of the inhibitors in the producing Streptomyces spp.

We have isolated a strain of Streptomyces exfoliatus producing extracellular protease inhibitors, leupeptin and leupeptin analogues (Kim et al., 1992, 1993). Here we have characterized the extracellular proteases, and have elucidated their physiological roles in terms of mycelial growth, autolysis of mycelia after the stationary phase in submerged culture, and morphological differentiation of surface cultures. We have also shown the existence of a 
leupeptin-inactivating protein (LIP), and demonstrated the biosynthetic relationships between leupeptin, LIP and extracellular proteases. The cascade regulatory roles for the molecules are discussed in conjunction with the growth and autolysis of mycelia in submerged cultures, and morphological differentiation of surface cultures of S. exfoliatus SMF13. This is the first report on the roles of leupeptin, LIP and trypsin-like protease (TLP) in relation to morphological differentiation of mycelia.

\section{METHODS}

Micro-organisms and media. The micro-organism used was Streptomyces exfoliatus SMF13 (Kim et al., 1992). Stock culture medium consisted of $(\%, \mathrm{w} / \mathrm{v})$ : glucose, 1 ; peptone, $0 \cdot 2$; yeast extract, $0 \cdot 3$; beef extract, $0 \cdot 1$; and agar, $1 \cdot 8$. Seed culture medium contained $(\%, \mathrm{w} / \mathrm{v})$ : glucose, 3.0 ; soytone, 1.8 ; peptone, 0.3 ; and $\mathrm{CaCO}_{3}, 0.4$. The main culture medium consisted of $(\%$, $\mathrm{w} / \mathrm{v}$ ): glucose, 0.5 ; sodium caseinate, $1 ; \mathrm{KH}_{2} \mathrm{PO}_{4}, 0.025$; $\mathrm{K}_{2} \mathrm{HPO}_{4}, \quad 0.085 ; \quad \mathrm{MgSO}_{4} .7 \mathrm{H}_{2} \mathrm{O}, 0.03 ; \mathrm{NaCl}, 0.03$; $\mathrm{FeSO}_{4} .7 \mathrm{H}_{2} \mathrm{O}, 0.001 ; \mathrm{CuSO}_{4} .5 \mathrm{H}_{2} \mathrm{O}, 0.001 ; \mathrm{CaCl}_{2} .2 \mathrm{H}_{2} \mathrm{O}$, 0.001 ; and $\mathrm{MnCl}_{2} \cdot 4 \mathrm{H}_{2} \mathrm{O}, 0.0003$. The phosphate and each of the salts were separately sterilized by membrane filtration $(0.2 \mu \mathrm{m}$, Millipore).

Strain maintenance and culture conditions. The strain was transferred to slopes of stock culture medium each month, and stored at $4{ }^{\circ} \mathrm{C}$. Spores from the stock cultures were suspended in saline solution and filtered through cotton (Hopwood et al., 1985). The spore suspension was kept at $-20^{\circ} \mathrm{C}$. For surface cultures, about $10^{3}$ spores were inoculated evenly on agar plates of the main culture medium, and incubated at $28^{\circ} \mathrm{C}$. For submerged batch cultures, the spore suspension was used to inoculate $50 \mathrm{ml}$ of seed culture medium (to $10^{6}$ spores $\mathrm{ml}^{-1}$ ) in a $500 \mathrm{ml}$ baffled flask, and cultured at $28^{\circ} \mathrm{C}$ for $36 \mathrm{~h}$ in a rotary shaking incubator (200 r.p.m.). The seed culture was used to inoculate $(5 \%, v / v) 3$ lof the main culture medium contained in a jar fermenter $\left(51\right.$; Korea Fermentor) maintained at $28^{\circ} \mathrm{C}$; the initial $\mathrm{pH}$ was adjusted to $7 \cdot 0$. Agitation and aeration were 200 r.p.m. and $1 \mathrm{v} \mathrm{v}^{-1} \mathrm{~min}^{-1}$, respectively.

Analysis of growth, glucose and ammonium ion in submerged cultures. Mycelia in submerged cultures were harvested aseptically by centrifugation at $10000 \mathrm{~g}$ for $10 \mathrm{~min}$, washed twice with physiological saline solution and once with distilled water, and then collected by vacuum filtration (Whatman filter paper GF/C) and dried at $80^{\circ} \mathrm{C}$ for $24 \mathrm{~h}$ for measurements of growth. The concentration of glucose was measured with dinitrosalicylic acid (Miller, 1959). The concentration of ammonium was measured with a specific ion analyser (model EA940, Orion Research).

Assay of proteases, leupeptin and LIP. Broth of submerged cultures was centrifuged at $10000 \mathrm{~g}$ for $10 \mathrm{~min}$ and the activities of proteases, leupeptin and LIP in the supernatant were measured. An agar plug $(5 \mathrm{~cm} \times 5 \mathrm{~cm})$ containing colonies was removed from the centre of each plate, homogenized in $10 \mathrm{ml}$ Tris/ $\mathrm{HCl}$ buffer $(0 \cdot 1 \mathrm{M}, \mathrm{pH} 7.5)$ and centrifuged $(10000 \mathrm{~g}$ for $10 \mathrm{~min})$. The activities of extracellular proteases and leupeptin in the supernatant were measured.

Total protease activity was assayed by measuring the concentration of tyrosine liberated from Hammarsten casein (Merck) after $15 \mathrm{~min}$ at $37^{\circ} \mathrm{C}$ and $\mathrm{pH} 7.5$ (Tris/ $\mathrm{HCl}$ buffer, $0 \cdot 1 \mathrm{M})$. The rate of hydrolysis of Hammarsten casein was linear during the $15 \mathrm{~min}$. One unit of casein hydrolytic activity (caseinase) was defined as the amount of enzyme needed to give $1 \mu \mathrm{g}$ tyrosine equivalent $\mathrm{min}^{-1}$ (Narahashi, 1970). Hydrolysis of synthetic substrates was assayed by measuring the amount of $p$ nitroanilides liberated from $N$-benzoyl-tyrosine $p$-nitroanilide (BTPNA), $N$-benzoyl-arginine $p$-nitroanilide (BAPNA), $p$-GluPhe-Leu $p$-nitroanilide (PFLNA), $N$-succinyl-Gly-Gly-Phe $p$ nitroanilide, $N$-succinyl-Tyr-Leu-Val $p$-nitroanilide and $N$ succinyl-Ala-Ala-Val $p$-nitroanilide. Enzyme reactions were carried out with $200 \mu \mathrm{M}$ aminoacyl $p$-nitroanilides at $35^{\circ} \mathrm{C}$ and $\mathrm{pH} 7.5$ (Tris/ $\mathrm{HCl}$ buffer, $0 \cdot 1 \mathrm{M}$ ). Activity was calculated from the linear part of the curve, using $\varepsilon_{405}=9620 \mathrm{~mol}^{-1} \mathrm{~cm}^{-1}$. One unit of hydrolytic activity was defined as the amount of enzyme needed to produce $1 \mu \mathrm{mol} p$-nitroanilide $\min ^{-1}$ (Sarath et al., 1989).

The effects of protease inhibitors on the hydrolytic activities of synthetic substrates were measured as follows: inhibitors such as leupeptin, antipain, $N \alpha$-p-tosyl-L-lysyl chloromethyl ketone (TLCK), N-tosyl-L-phenylalanine chloromethyl ketone (TPCK), chymostatin, EDTA, E-64 and pepstatin were preincubated with the protease solution for $5 \mathrm{~min}$. The activity remaining against BTPNA, BAPNA and $p$-Glu-Phe-Leu $p$ nitroanilide was measured. The activity of protease inhibitors is given by percentage inhibition $=100\{(A-B) / A\}$, where $A$ is protease activity without the inhibitor and $B$ is protease activity with the inhibitor (Aoyagi et al., 1969). The concentration of leupeptin in culture broth was calculated from a standard curve prepared with authentic leupeptin and $80 \mu \mathrm{g}$ papain as the target protease.

The activity of LIP was determined as follows : $1.0 \mathrm{ml}$ myceliumfree culture broth was preincubated with $300 \mu \mathrm{g}$ of leupeptin at $4{ }^{\circ} \mathrm{C}$ and $\mathrm{pH} 7.5$ (Tris/ $\mathrm{HCl}$ buffer, $0.1 \mathrm{M}$ ) for $1 \mathrm{~h}$ to compensate for the possible interaction between leupeptin and TLP. The preincubated reaction mixture was incubated at $35^{\circ} \mathrm{C}$ for $1 \mathrm{~h}$, then heated for $5 \mathrm{~min}$ at $80^{\circ} \mathrm{C}$ for complete inactivation of any protease and LIP in the reaction mixture. The remaining activity of leupeptin was assayed $(A)$. In parallel, the preincubated reaction mixture was heated at $80^{\circ} \mathrm{C}$ for $5 \mathrm{~min}$ for complete inactivation of LIP, then incubated at $35^{\circ} \mathrm{C}$ for $1 \mathrm{~h}$. The remaining activity of leupeptin was assayed $(B)$. The difference between $A$ and $B$ was defined as the leupeptin-inactivating activity. One unit of LIP was defined as the amount of enzyme needed for inactivation of $10 \mu \mathrm{g}$ leupeptin $\mathrm{min}^{-1}$.

Purification of proteases. Cell-free supernatant of the culture broth of $S$. exfoliatus SMF13 after centrifugation at $10000 \mathrm{~g}$ for 15 min was used as starting material for the purification of extracellular proteases. TLP was purified by DEAE-Sephadex A-50, hydroxyapatite and CM-Sephadex C-50 chromatography. Metalloprotease (MTP) was partially purified by DEAESephadex A-50 chromatography and gel filtration using Sephadex G-75. CTP was partially purified by DEAE-Sephadex A-50 and hydroxyapatite chromatography.

Additional experiments. Various protease inhibitors such as TPCK, EDTA and leupeptin were added to the culture broth. EDTA and leupeptin were dissolved in distilled water; TPCK was dissolved in ethanol, because it is not water-soluble. The protease inhibitor solutions were sterilized by filtration $(0.2 \mu \mathrm{m}$, Millipore) and added to culture broth to 0.1 or $0.2 \mathrm{mM}$. Various amounts $(0.1,0.2$ or $0.4 \mathrm{mg})$ of leupeptin were also added aseptically to the top of colonies developed on agar pieces. In the addition of glucose, sterile glucose solution was added to the culture broth to $100 \mathrm{mM}$.

Analysis of fermentation kinetic parameters. Kinetic parameters in batch culture were calculated as suggested by Pirt (1975). Specific mycelium growth rate $(\mu)$ was calculated by $\mu=(\mathrm{d} x / \mathrm{d} t) / x$, where $\mathrm{d} x / \mathrm{d} t$ is the differential change in concentration of biomass $\left(\mathrm{g} \mathrm{l}^{-1} \mathrm{~h}^{-1}\right)$ and $x$ is the concentration of biomass $\left(\mathrm{g} \mathrm{l}^{-1}\right)$. The specific glucose uptake rate $\left(q_{\mathrm{glc}}\right)$ was 
calculated by $q_{\mathrm{glc}}=(\mathrm{d}[\mathrm{glc}] / \mathrm{d} t) / x$, where $\mathrm{d}[\mathrm{glc}] / \mathrm{d} t$ is the differential change in concentration of glucose $\left(\mathrm{g} \mathrm{l}^{-1} \mathrm{~h}^{-1}\right)$ and $x$ is the concentration of biomass $\left(\mathrm{g}^{-1}\right)$. Kinetics parameters were calculated with data from the exponential growth phase.

Chemicals, reagents and reproducibility. All synthetic substrates, enzyme inhibitors and resins for protease purification were purchased from Sigma. All other chemicals were of reagent grade. Experiments were done in triplicate and mean values are given.

\section{RESULTS}

\section{Characteristics of extracellular proteases produced in submerged culture}

Mycelial growth, production of leupeptin and extracellular protease in batch culture containing sodium caseinate or casamino acids as sole nitrogen source are compared in Fig. 1. In the culture with sodium caseinate, a significant amount of extracellular protease was produced. Mycelial growth stopped at $46 \mathrm{~h}$ with exhaustion of glucose, and biomass declined without a distinctive stationary phase. Leupeptin production was closely related to mycelial growth, but leupeptin was completely degraded in the decline phase (Fig. 1a). In cultures with casamino acids, leupeptin production was associated with mycelial growth, but extracellular protease was not produced. The leupeptin concentration and biomass did not decline, but remained at their maximum values until the end of the batch culture (Fig. 1b).

The characteristics of the extracellular protease produced in the culture containing sodium caseinate were evaluated by determining its substrate specificity, and its response to various protease inhibitors (Table 1). It hydrolysed BAPNA, BTPNA, and PFLNA, but not $N$-succinyl-GlyGly-Phe $p$-nitroanilide, $N$-succinyl-Tyr-Leu-Val $p$-nitroanilide and $N$-succinyl-Ala-Ala-Val $p$-nitroanilide. The profiles of inhibition specificity varied; the BAPNAhydrolysing activity was completely inhibited by leupeptin, antipain and TLCK, and the PFLNA-hydrolysing activity was completely inhibited by EDTA, whereas the BTPNA-hydrolysing activity was inhibited about $80 \%$ by TPCK and chymostatin.

These results indicated that $S$. exfoliatus SMF13 produced at least three types of extracellular proteases. The protease active on BAPNA was a TLP, the protease active on BTPNA was a chymotrypsin-like protease (CTP) and the protease active on PFLNA was an MTP. The TLP was purified (molecular mass $31.8 \mathrm{kDa}$ ), and its characteristics were compared with those of CTP and MTP which were partially purified. TLP activity was optimum at $\mathrm{pH} 7 \cdot 5$, whereas CTP and MTP were more active at $\mathrm{pH} 9 \cdot 0$. The optimum temperature for TLP was $35-40^{\circ} \mathrm{C}$, whereas, that of MTP or CTP was $45^{\circ} \mathrm{C} . \mathrm{Co}^{2+}$ and $\mathrm{Ca}^{2+}$ activated MTP.

\section{Dynamic changes of leupeptin, CTP, MTP and TLP activities in submerged culture}

Changes in the activities of leupeptin, CTP, MTP and TLP during a batch culture containing sodium caseinate are shown in Fig. 2. The activity of CTP increased from the early exponential growth phase and that of MTP (a)

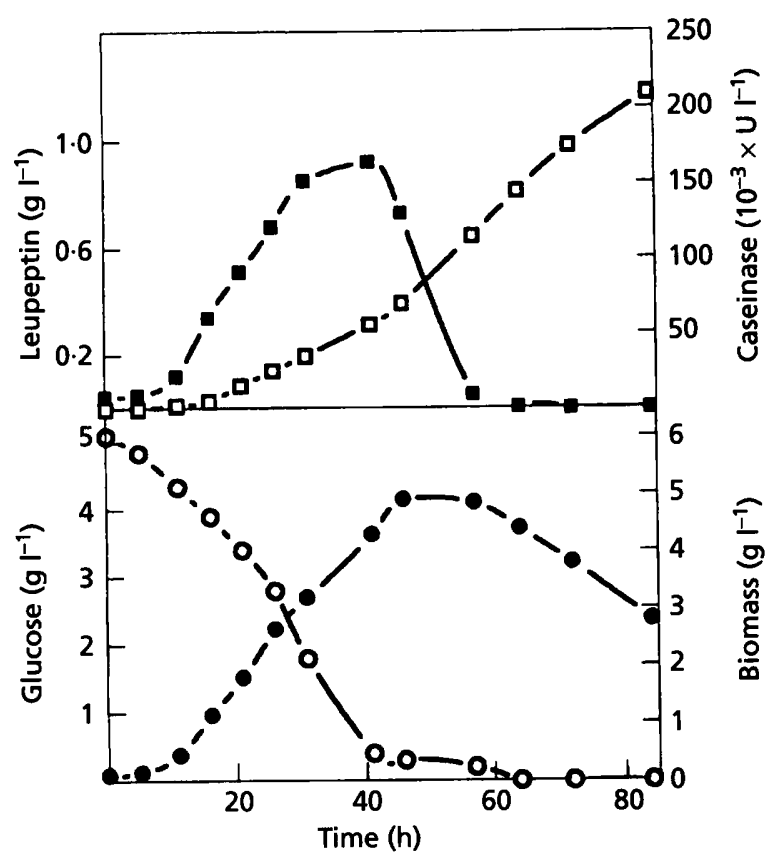

(b)

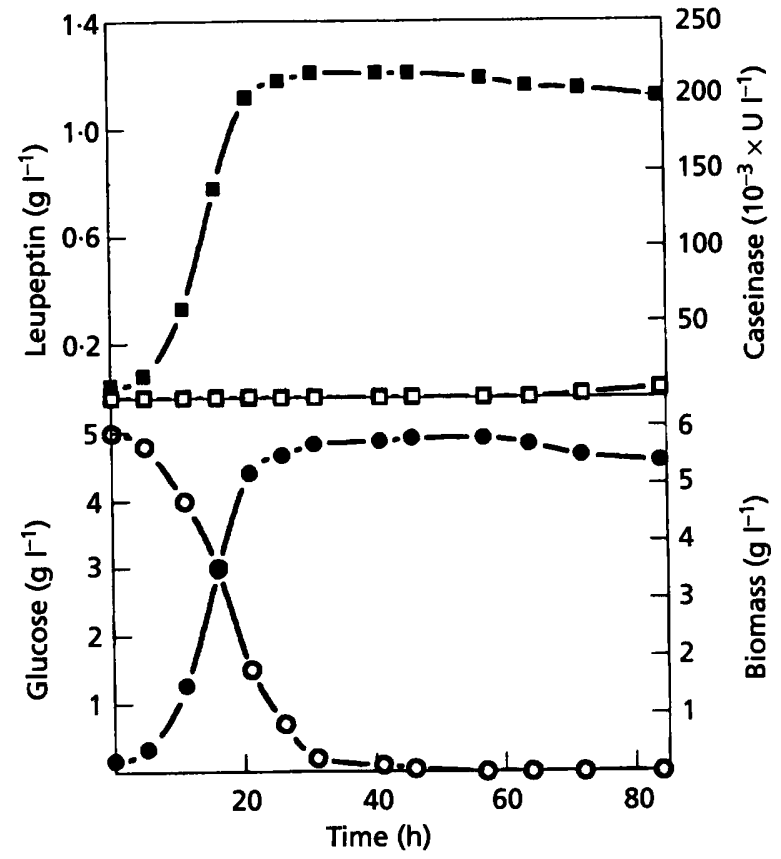

Fig. 1. Changes in the concentrations of glucose (O), biomass (O), caseinase $(\square)$ and leupeptin ( $\square$ ) in submerged batch cultures of $S$. exfoliatus SMF13. The cultures were grown in a chemically defined medium containing sodium caseinate (a) or casamino acids (b) as sole nitrogen source. 
Table 1. Effect of protease inhibitors on hydrolysis of synthetic substrates by broth from 4-d-old cultures of S. exfoliatus SMF13

The protease inhibitor was mixed with $100 \mu \mathrm{l}$ cell-free broth from 4-d-old cultures. After preincubation for 5 min, rates of BAPNA, BTPNA and PFLNA hydrolysis were measured.

\begin{tabular}{|c|c|c|c|c|c|c|c|c|c|c|}
\hline \multirow[t]{2}{*}{ Substrate } & \multicolumn{10}{|c|}{$\begin{array}{l}\text { Hydrolytic activity }\left(\mathrm{U} \mathrm{ml}^{-1}\right) \text { and percentage inhibition } \\
\text { (in parentheses) in presence of inhibitors: }\end{array}$} \\
\hline & Control & $\begin{array}{l}\text { Leupeptin* } \\
(50 \mu \mathrm{M})\end{array}$ & $\begin{array}{l}\text { Leupeptin } \\
(50 \mu \mathrm{M})\end{array}$ & $\begin{array}{c}\text { Antipain } \\
(50 \mu M)\end{array}$ & $\begin{array}{l}\text { Chymostatin } \\
\qquad(50 \mu \mathrm{M})\end{array}$ & $\begin{array}{c}\text { TLCK } \\
(50 \mu \mathrm{M})\end{array}$ & $\begin{array}{c}\text { TPCK } \\
(50 \mu \mathrm{M})\end{array}$ & $\begin{array}{c}E-64 \\
(10 \mu \mathrm{M})\end{array}$ & $\begin{array}{l}\text { EDTA } \\
(1 \mathrm{mM})\end{array}$ & $\begin{array}{c}\text { Pepstatin } \\
(50 \mu \mathrm{M})\end{array}$ \\
\hline BAPNA & 0.410 & $\begin{array}{l}0 \cdot 002 \\
(99 \cdot 5)\end{array}$ & $\begin{array}{l}0 \cdot 003 \\
(99 \cdot 3)\end{array}$ & $\begin{array}{l}0 \cdot 002 \\
(99 \cdot 5)\end{array}$ & $\begin{array}{l}0 \cdot 080 \\
(80 \cdot 5)\end{array}$ & $\begin{array}{l}0 \cdot 028 \\
(93 \cdot 1)\end{array}$ & $\begin{array}{l}0 \cdot 408 \\
(0 \cdot 5)\end{array}$ & $\begin{array}{l}0 \cdot 402 \\
(2 \cdot 0)\end{array}$ & $\begin{array}{l}0 \cdot 381 \\
(7 \cdot 0)\end{array}$ & $\begin{array}{l}0 \cdot 415 \\
(0 \cdot 0)\end{array}$ \\
\hline BTPNA & $0 \cdot 612$ & $\begin{array}{l}0.601 \\
(1 \cdot 8)\end{array}$ & $\begin{array}{l}0 \cdot 605 \\
(1 \cdot 1)\end{array}$ & $\begin{array}{l}0.627 \\
(0.0)\end{array}$ & $\begin{array}{c}0.163 \\
(73 \cdot 4)\end{array}$ & $\begin{array}{l}0.692 \\
(0.0)\end{array}$ & $\begin{array}{l}0 \cdot 140 \\
(77 \cdot 1)\end{array}$ & $\begin{array}{l}0.693 \\
(0.0)\end{array}$ & $\begin{array}{l}0.561 \\
(8 \cdot 3)\end{array}$ & $\begin{array}{l}0.641 \\
(0 \cdot 0)\end{array}$ \\
\hline PFLNA & 0.223 & $\begin{array}{l}0 \cdot 216 \\
(3 \cdot 1)\end{array}$ & $\begin{array}{l}0 \cdot 218 \\
(2 \cdot 2)\end{array}$ & $\begin{array}{l}0.228 \\
(0 \cdot 0)\end{array}$ & $\begin{array}{c}0.150 \\
(32 \cdot 7)\end{array}$ & $\begin{array}{l}0.236 \\
(0 \cdot 0)\end{array}$ & $\begin{array}{c}0 \cdot 168 \\
(24 \cdot 7)\end{array}$ & $\begin{array}{l}0 \cdot 209 \\
(6 \cdot 3)\end{array}$ & $\begin{array}{c}0.006 \\
(97 \cdot 3)\end{array}$ & $\begin{array}{l}0 \cdot 207 \\
(7 \cdot 2)\end{array}$ \\
\hline
\end{tabular}

* Leupeptin purified from the culture broth of $S$. exfoliatus SMF13.

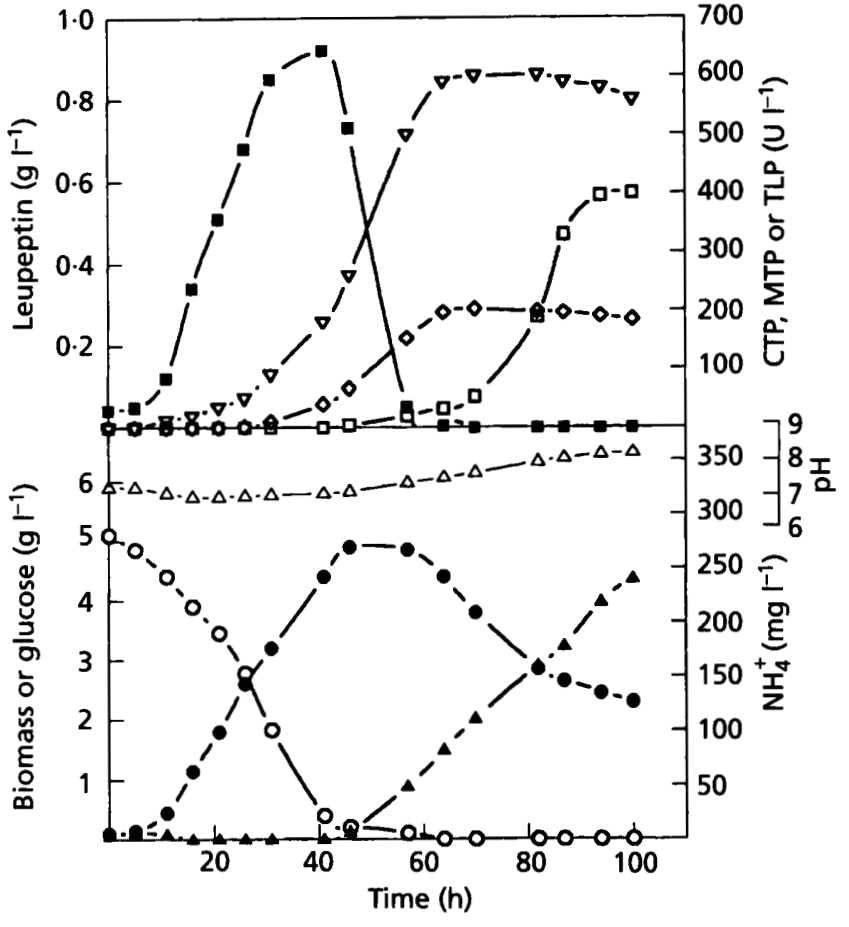

Fig. 2. Changes in $\mathrm{pH}(\triangle)$ and in the concentrations of glucose $(0)$, biomass (O), ammonium ions ( $\boldsymbol{\Lambda})$, leupeptin $(\square)$, CTP $(\nabla)$, MTP $(\diamond)$ and TLP $(\square)$ in submerged batch cultures of $S$. exfoliatus SMF13. Cultures were grown in a chemically defined medium containing sodium caseinate as the sole nitrogen source.

increased from the late exponential growth phase. Both activities were at their maxima during the stationary phase. However, the activity of TLP started to rise only at the beginning of the stationary phase. The increase in the activity of TLP correlated with the inactivation of leupeptin, which was produced in association with

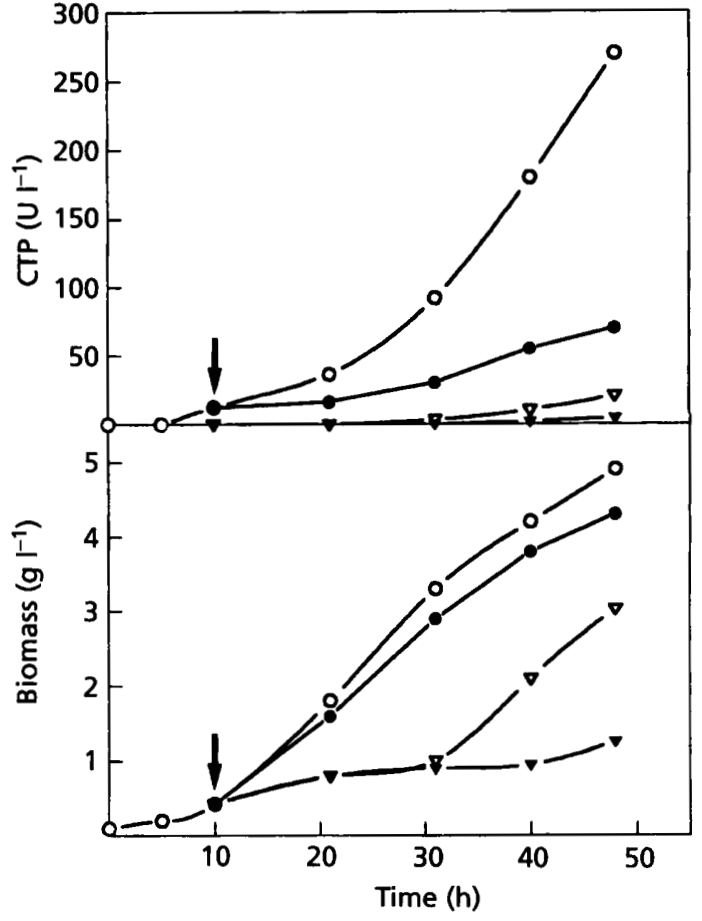

Fig. 3. Effect of addition of TPCK on CTP activity and growth of $S$. exfoliatus SMF13. Cultures were grown in a chemically defined medium containing sodium caseinate as the sole nitrogen source. TPCK in ethanol $[0.1 \mathrm{mM}(\nabla)$ and $0.2 \mathrm{mM}(\nabla)]$ was added to the culture in early exponential phase as indicated by the arrows. Changes in CTP activity and growth were compared with those in control cultures supplemented with saline (O) and ethanol (O).

mycelial growth. Moreover, the increase in TLP activity was linked to the decrease in biomass, the accumulation of ammonium ions, and the concomitant increase in culture $\mathrm{pH}$. These results suggested that TLP might degrade mycelium proteins. 


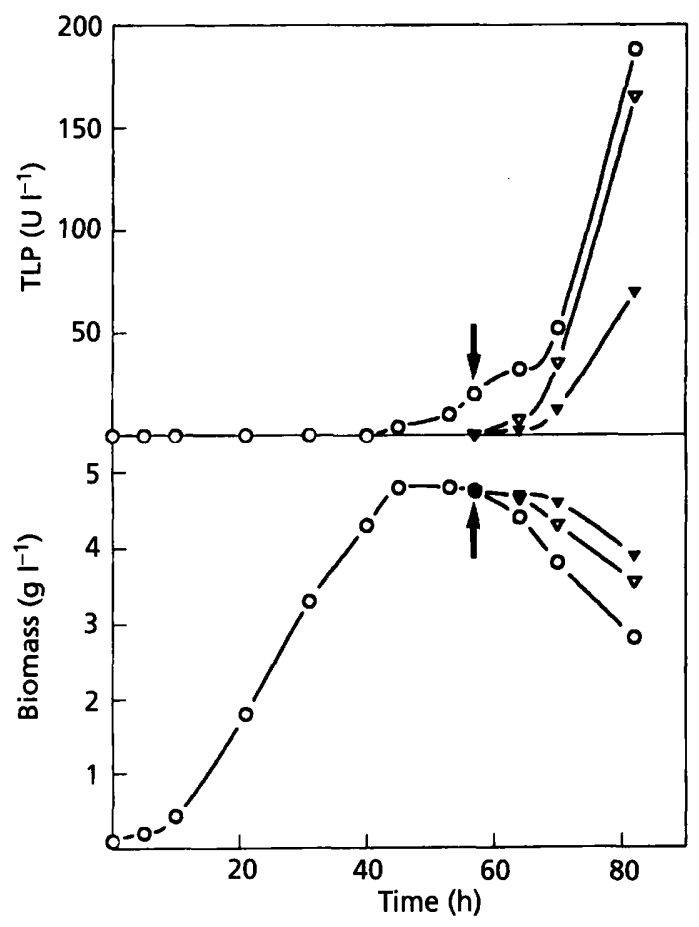

Fig. 4. Effect of addition of leupeptin on TLP activity and mycelium autolysis of $S$. exfoliatus SMF13. Cultures were grown in a chemically defined medium containing sodium caseinate as the sole nitrogen source. Leupeptin $[0.1 \mathrm{mM}(\nabla)$ and $0.2 \mathrm{mM}$ $(\nabla)$ ] in distilled water was added to the culture in early exponential phase as indicated by the arrows. Changes in TLP activity and biomass were compared with those in control cultures where no leupeptin was added (O).

Table 2. Kinetic parameters for S. exfoliatus SMF13 batch cultures at various concentrations of glucose

\begin{tabular}{|c|c|c|c|}
\hline \multirow[t]{2}{*}{ Kinetic parameters } & \multicolumn{3}{|c|}{$\begin{array}{c}\text { Glucose concentration } \\
\qquad\left(\mathrm{g}^{-1}\right)\end{array}$} \\
\hline & 5 & 10 & 30 \\
\hline Caseinase* $\left(10^{-3} \times \mathrm{U} \mathrm{l}^{-1}\right)$ & $250 \cdot 3$ & $175 \cdot 2$ & $20 \cdot 0$ \\
\hline Biomass* $\left(\mathrm{g} \mathrm{l}^{-1}\right)$ & $4 \cdot 9$ & $5 \cdot 4$ & 6.6 \\
\hline Specific growth ratef, $\mu\left(\mathrm{h}^{-1}\right)$ & 0.068 & 0.074 & 0.095 \\
\hline $\begin{array}{l}\text { Specific glucose uptake rate } \\
q_{\mathrm{gl|}}\left(\mathrm{g} \mathrm{g}^{-1} \mathrm{~h}^{-1}\right)\end{array}$ & 0.062 & $0 \cdot 117$ & 0.202 \\
\hline $\begin{array}{l}\text { Maximum biomass yield } \\
Y_{x / \mathrm{gle}}\left(\mathrm{g} \mathrm{g}^{-1}\right)\end{array}$ & $1 \cdot 101$ & 0.635 & $0 \cdot 470$ \\
\hline
\end{tabular}

* Maximum concentration of caseinase and biomass in $72 \mathrm{~h}$ cultures. † Maximum values calculated from data obtained during exponential growth.

The late appearance of MTP and TLP in the culture supernatant is not due to the release of these compounds by autolysis. Addition of kanamycin, a translation inhibitor, immediately stopped the production of MTP and TLP (data not shown), demonstrating that the appearance of these compounds resulted from the rapid export of products of de novo protein synthesis, rather than secretion of compounds that had accumulated intracellularly.

\section{Addition of protease inhibitors and changes of mycelial growth pattern in submerged culture}

Effects of protease inhibitors such as TPCK, EDTA and leupeptin on mycelial growth and the activities of CTP, MTP and TLP in submerged culture were determined by the addition of the inhibitors to cultures during early exponential growth. Addition of EDTA significantly inhibited MTP activity, but only slightly inhibited mycelial growth (data not shown). Addition of leupeptin did not inhibit mycelial growth (data not shown). However, TPCK, the specific inhibitor of CTP, inhibited mycelial growth and CTP activity in cultures containing sodium caseinate as the sole nitrogen source (Fig. 3). Mycelial growth was not inhibited by TPCK in the culture containing casamino acids as the sole nitrogen source, where CTP was not produced (data not shown). This suggested that TPCK inhibited only CTP activity, and that mycelial growth was retarded indirectly by the inhibition of CTP.

The effects of TPCK, EDTA and leupeptin on the autolysis of mycelia in submerged culture were compared by addition of the inhibitors to cultures in early stationary phase. The activities of CTP and MTP produced before the addition of TPCK and EDTA were significantly inhibited by the addition of TPCK and EDTA, respectively, whereas autolysis of mycelia was not affected (data not shown). However, autolysis of mycelia was clearly retarded by addition of leupeptin, the autogenous specific inhibitor of TLP (Fig. 4). Moreover, TLP activity in the culture broth was reduced to zero immediately after addition of leupeptin, but returned after an extended lag which was proportional to the amount of leupeptin added. Autolysis of the mycelia resumed simultaneously with the restoration of TLP activity which was accompanied by inactivation of leupeptin, suggesting the presence of a leupeptin-inactivating substance.

To verify the existence of a leupeptin-inactivating substance, the activity was partially purified by steps such as ammonium sulphate fractionation, DEAE-Sephadex chromatography and gel-permeation chromatography using Sephadex G-75. The product then inactivated leupeptin with about 70-times higher specific activity than the cell-free culture supernatant. Also, heat-treated leupeptin-inactivating substance did not inactivate leupeptin (data not shown). This suggested that the leupeptininactivating substance was a type of protein, which we termed LIP.

\section{Effects of glucose on the production of leupeptin, CTP, MTP and TLP in submerged culture}

Since leupeptin, CTP, MTP and TLP were produced sequentially in batch cultures containing sodium caseinate (Fig. 1), various medium components were evaluated for their role in production of these molecules. The concentration of glucose was particularly important. Therefore, the effects of the initial glucose concentration on the 

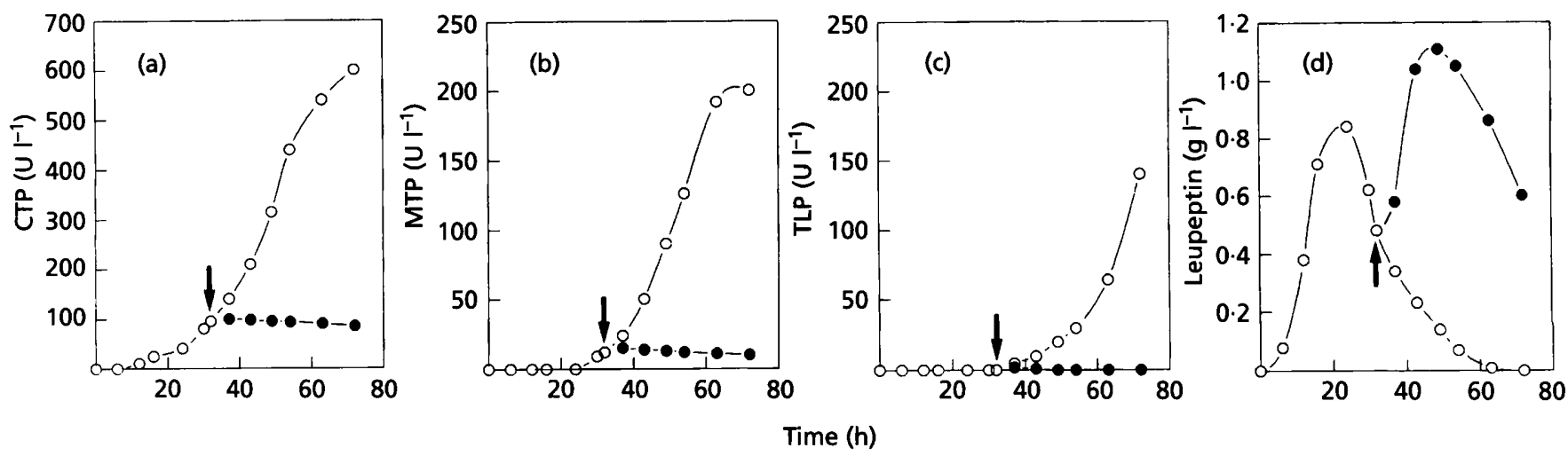

Fig. 5. Effect of addition of glucose on the production of CTP (a), MTP (b), TLP (c) and leupeptin (d) in batch cultures of $S$. exfoliatus SMF13. Cultures were grown in a chemically defined medium containing sodium caseinate as sole nitrogen source. Arrows indicate when $100 \mathrm{mM}$ glucose $(O)$ or saline $(O)$ were added to the culture broths during early stationary phase.

(a)

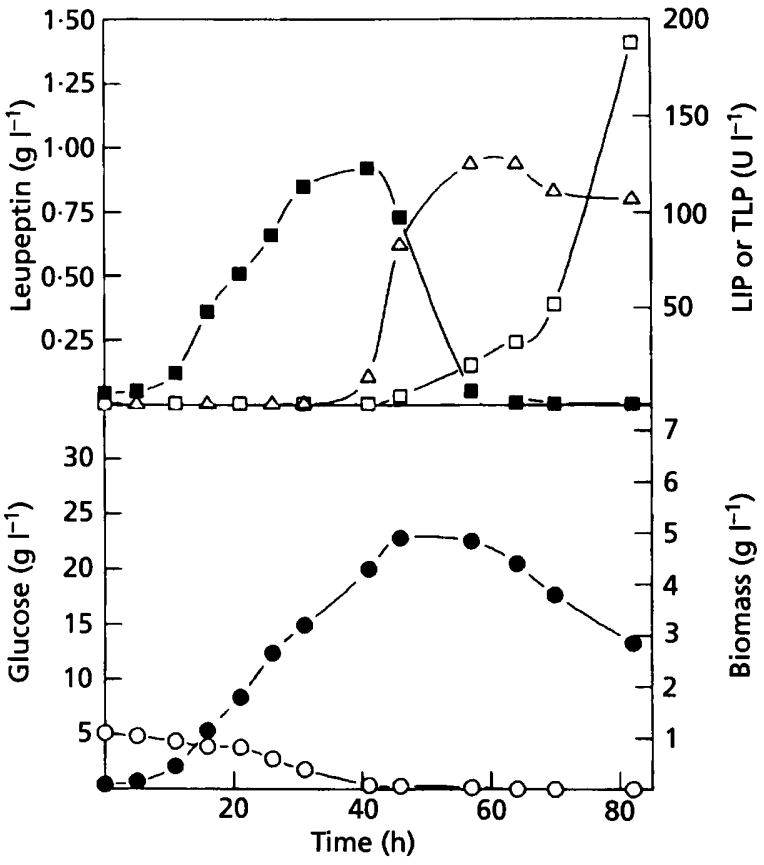

(b)

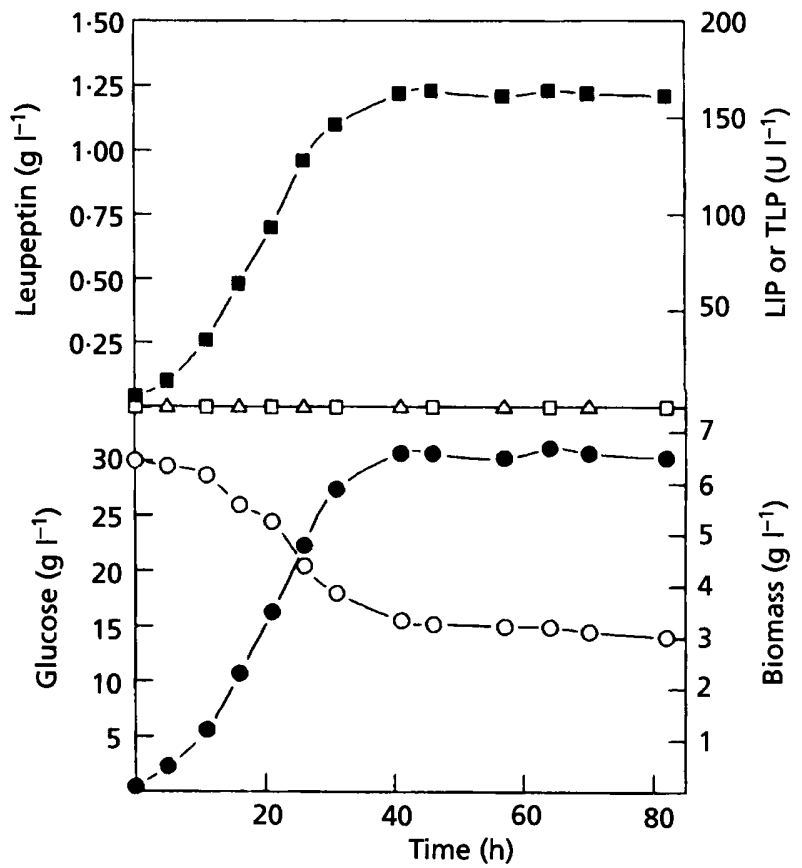

Fig. 6. Production of leupeptin ( $\square)$, LIP $(\triangle)$ and TLP $(\square)$ in glucose-limited (a) and glucose-sufficient (b) submerged cultures of $S$. exfoliatus SMF13. Cultures were grown in a chemically defined medium containing sodium caseinate as sole nitrogen source. Mycelial growth $(\odot)$ and utilization of glucose $(O)$ were compared.

production of extracellular protease and on mycelial growth in batch cultures containing sodium caseinate were evaluated (Table 2). Higher values for specific growth rate $(\mu)$ and specific glucose uptake rate $\left(q_{\mathrm{glc}}\right)$ were obtained from cultures with the higher initial concentrations of glucose. However, production of extracellular protease was higher at the lower of glucose concentrations. The mycelial growth yields $\left(Y_{x / \text { glc }}\right)$ were also higher at lower concentrations of glucose.

The effects of addition of glucose $(100 \mathrm{mM})$ to cultures in mid-exponential growth are shown in Fig. 5(a-d).
Production of CTP, MTP and TLP ceased, but leupeptin production increased. These results suggested that the regulatory role of glucose on the biosynthesis of CTP, MTP and TLP might be different from that on the biosynthesis of leupeptin.

\section{Relationships between growth and production of leupeptin, LIP and TLP in submerged culture}

Changes in growth, and the concentrations of leupeptin, LIP and TLP during batch culture were compared in glucose-limited and glucose-sufficient batch cultures. In 
the glucose-limited cultures, mycelial growth turned to stationary phase when glucose was completely utilized, and the culture immediately entered the death phase (Fig. 6a). Leupeptin was produced during mycelial growth, but was inactivated with the rapid rise of LIP activity. The decrease in the concentration of leupeptin in turn gave rise to increased TLP activity. LIP and TLP started to be produced in the decelerating growth phase, with LIP being produced more rapidly than TLP.

In glucose-sufficient conditions, stationary phase was reached while the residual glucose concentration was high and stationary phase was maintained for an extended period. Leupeptin was produced in close association with mycelial growth, and the concentration of leupeptin was maintained throughout stationary phase. (Fig. 6b). Neither LIP nor TLP were produced in glucose-sufficient conditions.

Although the maximum concentration of biomass in the glucose-sufficient condition was higher than in the carbon-limited condition, the maximum growth yield $\left(Y_{x / \mathrm{glc}}\right)$ in the glucose-limited culture was much higher $\left(1.101\right.$ vs $\left.0.470 \mathrm{~g} \mathrm{~g}^{-1}\right)$. Mycelial growth and leupeptin production were stimulated by excess glucose, whereas LIP and TLP production were clearly enhanced by limiting glucose. Therefore, production of LIP and TLP might be closely related to the autolysis of mycelium in the glucose-limited cultures.

\section{Relationship between colony develpment and production of leupeptin, CTP, MTP and TLP in the surface culture}

The production of leupeptin and extracellular proteases was measured during colony development in surface cultures of S. exfoliatus SMF13 (Fig. 7). Leupeptin was produced during the growth of substrate mycelia but stopped after $24 \mathrm{~h}$ and began to decrease before the onset of the aerial mycelium formation, and its activity had completely disappeared when aerial mycelium formation was very active.

Production of CTP started with the formation of substrate mycelia and continued to the stage of aerial mycelium formation. The activity of CTP gradually decreased during sporulation. However, production of MTP started in the middle of substrate mycelium formation and rapidly increased at the onset of the aerial mycelium formation; the activity was at a maximum during sporulation. The production of TLP started just before the onset of aerial mycelium formation, and was closely related to the inactivation of leupeptin (Fig. 7).

\section{Effect of addition of leupeptin on aerial mycelium formation in surface cultures}

The effect of external addition of leupeptin on the morphological differentiation of $S$. exfoliatus SMF13 in surface cultures was measured. When leupeptin $(0.4 \mathrm{mg}$

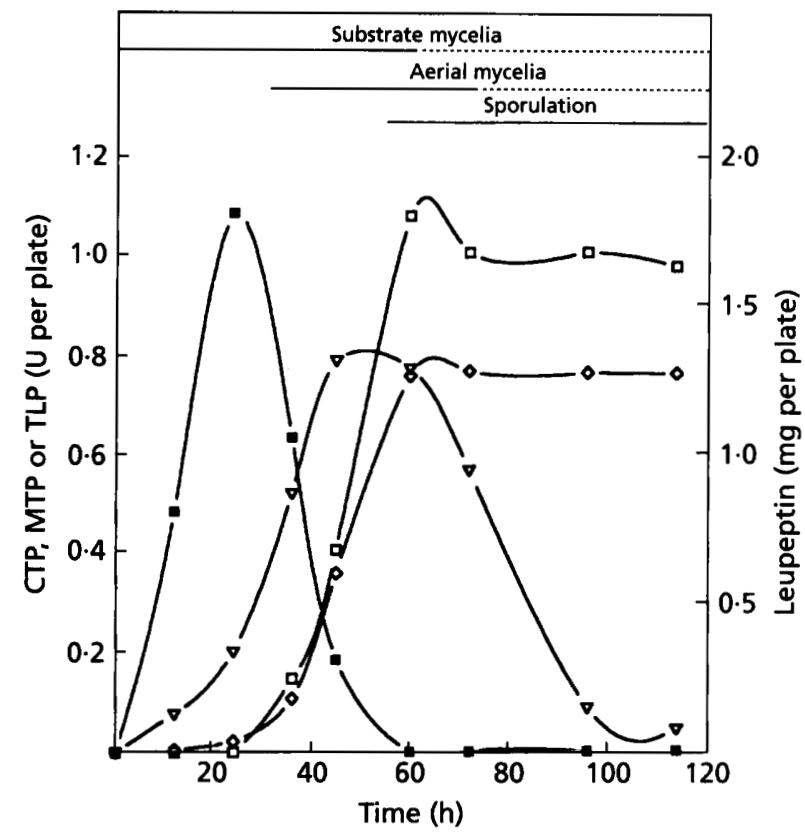

Fig. 7. Relationships between morphological differentiation of mycelium and production of leupeptin $(\square)$, CTP $(\nabla), \operatorname{MTP}(\diamond)$ and TLP $(\square)$ in surface cultures of $S$. exfoliatus SMF13. Cultures were grown in a chemically defined medium containing sodium caseinate and glucose.

(a)

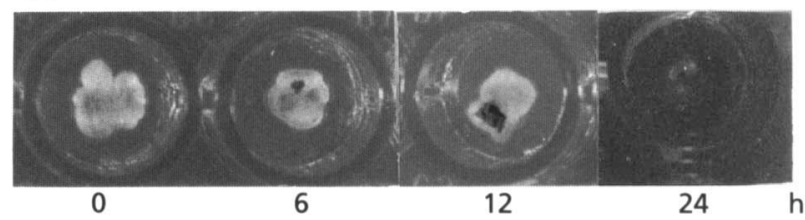

(b)

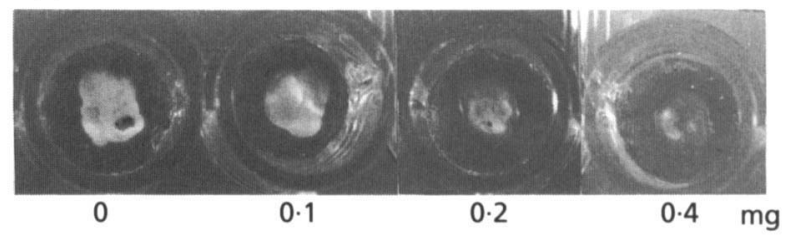

Fig. 8. Effects of time of addition (a) and concentration (b) of leupeptin on the retardation of aerial mycelium formation in surface cultures of S. exfoliatus SMF13. Cultures were grown in a chemically defined medium containing sodium caseinate and glucose. Leupeptin $(0.4 \mathrm{mg})$ was added to the top surface of colonies at the times indicated. (b) Leupeptin (as indicated) was added to the top surface of colonies at $24 \mathrm{~h}$, just before the onset of aerial mycelium formation. Colonies were photographed after $36 \mathrm{~h}$.

per colony) was added externally to the top surface of a colony at different times, a significant retardation in aerial mycelium formation was observed in $24 \mathrm{~h}$ cultures, but not in 0,6 and $12 \mathrm{~h}$ cultures (Fig. 8a). The effect of addition of increasing amounts of leupeptin $(0,0 \cdot 1,0 \cdot 2$ or 
$0.4 \mathrm{mg}$ per colony) on the retardation of aerial mycelia formation were compared again by external addition at $24 \mathrm{~h}$ (Fig. 8b). The retardation of aerial mycelium formation was closely related to the concentration of leupeptin added externally. Furthermore, formation of aerial mycelia was restored after an interval that was proportional to the amount of leupeptin added. The interval was about 2,4 or $8 \mathrm{~h}$ when $0 \cdot 1,0.2$ or $0.4 \mathrm{mg}$ leupeptin per colony was added, respectively.

Our preliminary experiment also showed that the production of TLP and the inactivation of leupeptin started from the centre of the colony, which might be nutrientlimited, and that leupeptin was largely produced in the peripheral regions where growth of substrate mycelia was active. Aerial mycelia started to form from the centre of the colony (data not shown).

\section{DISCUSSION}

S. exfoliatus SMF13 produced extacellular proteases (CTP, MTP and TLP) and a protease inhibitor (leupeptin) in batch cultures containing sodium caseinate, the mycelium and leupeptin being degraded when glucose was depleted. However, no extracellular protease was produced in cultures containing casamino acids as the sole nitrogen source; in these cultures, mycelium and leupeptin remained intact throughout the period of study.

CTP production was closely linked to mycelial growth and addition of TPCK, the specific inhibitor of CTP, reduced both the activity of CTP and mycelium growth. The biomass production yield value $\left(Y_{x / \mathrm{glc}}\right)$ increased when glucose was limiting. The simplest interpretation of these observations is that CTP participates primarily in the utilization of proteinaceous nitrogen for growth, and that sodium caseinate is used not only as a nitrogen source but also as carbon and energy sources when glucose is limiting.

TLP was produced in the stationary phase when glucose was completely exhausted. The increase in TLP activity was linked to the decrease in biomass and the accumulation of ammonium ions in the culture broth. Addition of leupeptin, the autogenous inhibitor of TLP, not only inhibited TLP activity, but also retarded hydrolysis of the mycelia in the decline phase. More interestingly, leupeptin was produced in association with mycelial growth, but it was stable in cultures with excess glucose, and inactivated rapidly in glucose-limited cultures. The inactivation of leupeptin in glucose-limited cultures was accompanied by the production of LIP which started earlier than that of TLP. LIP was produced only in glucose-limited cultures. The results indicate that leupeptin, LIP and TLP may play important roles in the regulation of mycelial growth and the hydrolysis of mycelium. Leupeptin regulates the activity of TLP, LIP inactivates leupeptin when TLP activity is required, and TLP functions as an enzyme involved in the hydrolysis of mycelial protein.

The growth of streptomycetes on solid media progresses through the sequential formation of substrate mycelium and aerial hyphae (Wildermath, 1970). The aerial hyphae appear to grow at least partially by utilization of degraded substrate mycelia (Mendez et al., 1985), because the aerial mycelia have little access to other sources of nutrients (Chater \& Merrick, 1979; Chater, 1984). Leupeptin production was closely related to the formation of substrate mycelium; leupeptin activity started to decrease before the onset of aerial mycelium formation, and was completely inactivated during aerial mycelium formation. Production of TLP started just before the onset of aerial mycelium formation, and the formation of aerial mycelia in surface cultures was retarded by addition of leupeptin. These results imply that leupeptin produced during substrate mycelium formation had to be inactivated before (or whilst) aerial mycelia were formed, and that TLP may play an important role in utilization of substrate mycelium protein for aerial mycelium formation. It may reasonably be assumed that leupeptin protects growing mycelia from the hydrolytic invasion of TLP which is produced in neighbouring growth-limited mycelia. For the expression of TLP activity, the leupeptin accumulated in the culture must be inactivated. This is accomplished by LIP, which is induced when the carbon and energy source is exhausted. Consequently, TLP is unlocked and starts to hydrolyse non-growing mycelia.

This is the first report on the identification of the roles of leupeptin, LIP and TLP in morphological changes. The regulatory actions of compounds that are closely related in mode of action, and are sequentially biosynthesized, may give selective advantages during colony development in natural soil environments. Although molecular regulatory mechanisms for the biosynthesis of the compounds have not yet been established, the results obtained here provide an insight into the morphological differentiation of Streptomyces spp.

\section{ACKNOWLEDGEMENTS}

We thank Professors Y. C. Hah and K. F. Chater for thoughtful discussions and revision of the manuscript. This work was supported by a research grant from the Research Center for Molecular Microbiology (RCMM) sponsored by the Korea Science and Engineering Foundation (KOSEF).

\section{REFERENCES}

Aoyagi, T. (1989). Protease inhibitor and biological control. In Bioactive Metabolites from Microorganisms: Progress in Industrial Microbiology, vol. 27, pp. 403-418. Edited by M. E. Bushell \& U. Gräfe. New York: Elsevier.

Aoyagi, T., Miyata, S., Nanbo, M., Kojima, F., Matsuzaki, M., Ishizuka, M., Takeuchi, T. \& Umezawa, H. (1969). Biological activities of leupeptins. J Antibiot 22, 558-568.

Aoyagi, T., Ishizuka, M., Takeuchi, T. \& Umezawa, H. (1977). Enzyme inhibitors in relation to cancer therapy. $J$ Antibiot $\mathbf{3 0}$, 121-132.

Bond, J. S. \& Butler, P. E. (1987). Intracellular proteases. Annu Rev Biochem 56, 333-364.

Bradiey, S. G. (1988). Actinomycete protease and their role in regulation. In Biology of Actinomycetes -'88, pp. 433-438. Edited by Y. Okami, T. Beppu \& H. Ogawara. Tokyo: Japan Scientific Societies Press. 
Chater, K. F. (1984). Morphological and physiological differentiation in Streptomyces. In Microbial Development, pp. 89-115. Edited by R. Losick \& L. Shapiro. Cold Spring Harbor, NY: Cold Spring Harbor Laboratory.

Chater, K. F. \& Merrick, M. J. (1979). Streptomyces. In Developmental Biology of Prokaryotes, pp. 93-114. Edited by J. H. Parish. Oxford: Blackwells.

Gibb, G. D. \& Strohl, W. R. (1988). Physiological regulation of protease activity in Streptomyces peucetius. Can J Microbiol 34, $187-190$.

Ginther, C. L. (1978). Sporulation and the production of serine protease and cephamycin C by Streptomyces lactamdurans. Antimicrob Agents Chemother 15, 522-526.

Gräfe. U. (1989). Autoregulatory secondary metabolite from actinomycetes. In Regulation of Secondary Metabolism in Actinomycetes, pp. 75-126. Edited by S. Shapiro. Boca Raton, FL: CRC Press.

Holzer, H. (1983). Regulation of proteinase in Saccharomyces cerevisiae. In Protease Inbibitors: Medical and Biological Aspects, pp. 181-190. Edited by N. Katunuma, H. Umezawa \& H. Holzer. Tokyo: Japan Scientific Society Press.

Hopwood, D. A., Bibb, M. J., Chater, K. F., Kieser, T., Bruton, C. J., Kieser, H. M., Lydiate, D. J., Smith, C. P., Ward, J. M. \& Schrempf, H. (1985). Genetic Manipulation of Streptomyces: a Laboratory Manual. Norwich: John Innes Foundation.

Kim, I. S., Kim, H. T., Ward, A. C., Goodfellow, M., Hah, Y. C. \& Lee, K. J. (1992). Numerical identification of a Streptomyces strain producing thiol protease inhibitor. J Microbiol Biotechnol 2, 220-225.

Kim, I. S., Han, Y. T., Barrow, K. D. \& Lee, K. J. (1993). The structure of protease inhibitors produced by Streptomyces exfoliatus SMF13. Korean J Microbiol 31, 326-334.

Mendez, C., Braña, A. F., Manzanal, M. B. \& Hardisson, C. (1985). Role of substrate mycelium in colony development in Streptomyces. Can J Microbiol 31, 446-450.

Miller, G. L. (1959). Use of dinitrosalicilic acid reagent for determination of reducing sugar. Anal Chem 31, 426-428.
Narahashi, Y. (1970). Pronase. Methods Enzymol 19, 651-664

Pirt, S. J. (1975). Parameters of growth and analysis of growth data. In Principles of Microbe and Cell Cultivation, pp. 4-14. Oxford: Blackwells.

Sarath, G., De La Motte, R. S. \& Wagner, F. W. (1989). Protease assay methods. In Proteolytic Enzymes: a Practical Approach, pp. 25-55. Edited by R. J. Beynone \& J. S. Bond. Oxford: IRL Press.

Shapiro, S. (1989). Nitrogen assimilation in actinomycetes and the influence of nitrogen nutrition on actinomycete secondary metabolism. In Regulation of Secondary Metabolism in Actinomycetes, pp. 149-153. Edited by S. Shapiro. Boca Raton, FL: CRC Press.

Suda, H., Aoyagi, T., Hamada, M., Takeuchi, T. \& Umezawa, H. (1972). Antipain, a new protease inhibitor isolated from actinomycetes. $J$ Antibiot 25, 263-266.

Umezawa, H. (1982). Low-molecular-weight enzyme inhibitors from microbial origin. Annu Rev Microbiol 36, 75-99.

Umezawa, H. (1988). Low-molecular-weight enzyme inhibitors and immunomodifiers. In Actinomycetes in Biotechnology, pp. 285-325. Edited by M. Goodfellow, S. T. Williams \& M. Mordarski. London: Academic Press.

Umezawa, H., Aoyagi, T., Morishima, H., Kunimoto, S., Matsuzaki, M., Hamada, M. \& Takeuchi, T. (1970). Chymostatin, a new chymotrypsin inhibitor produced by actinomycetes. $J$ Antibiot 23, 425-427.

Umezawa, H., Aoyagi, T., Okura, A., Morishima, H., Takeuchi, T. \& Okami, Y. (1973). Elastatinal, a new elastase inhibitor produced by actinomycetes. $J$ Antibiot 26, 787-789.

Wildermath, H. (1970). Development and organization of the aerial mycelium in Streptomyces coelicolor. J Gen Microbiol 60, 43-50.

Yuan, L. \& Cole, G. T. (1989). Characterization of a proteinase inhibitor isolated from the fungal pathogen Coccidioides immitis. Biochem J 257, 729-736.

Received 2 August 1994; revised 24 October 1994; accepted 7 November 1994. 